\title{
A near-IR spectrum of the DO white dwarf RE J0503-285^
}

\author{
P. D. Dobbie ${ }^{1}$, M. R. Burleigh ${ }^{1}$, A. J. Levan ${ }^{1}$, M. A. Barstow ${ }^{1}$, R. Napiwotzki ${ }^{1}$, and I. Hubeny ${ }^{2}$ \\ 1 Dept. of Physics \& Astronomy, University of Leicester, Leicester LE1 7RH, UK \\ e-mail: [pdd;mbu;anl;mab]@star.le.ac.uk \\ ${ }^{2}$ Dept. of Astronomy and Steward Observatory, University of Arizona, Tucson, AZ 85721, USA \\ e-mail: hubeny@as.arizona.edu
}

Received 8 April 2005 / Accepted 13 June 2005

\begin{abstract}
We present a near-IR spectroscopic analysis of the intriguing DO white dwarf RE J0503-285. The IR spectrum fails to reveal evidence of the presence of a spatially unresolved, cool, late-type companion. Hence we have placed an approximate limit on the spectral-type and mass of a putative companion (later than M8, $M<0.085 M_{\odot}$ ). This result rules out ongoing interaction between the white dwarf and a close companion with $M \geq 0.085 M_{\odot}$ as responsible for the discrepancies between the observed photospheric abundances and model predictions. As the possibility remains that there is a cooler companion lying beyond the detection threshold of this study we use our modelling to estimate the improvement in sensitivity offered by a Spitzer observation.
\end{abstract}

Key words. stars: white dwarfs - stars: atmospheres - stars: binaries: spectroscopic - stars: individual: RE J0503-285

\section{Introduction}

In accord with a theoretical prediction made several decades ago (Schatzman 1958), the atmospheres of white dwarfs are observed to be dominated either by hydrogen (DAs) or by helium (DO/DBs). Unimpeded, their high surface gravities lead to the settling out of heavier elements on timescales of mere days to months (e.g. Dupuis et al. 1993). In the standard theory of single star evolution the distinction between the two compositions is made at birth. If a star leaves the AGB during a period of quiescent hydrogen shell burning it evolves onto the hydrogen dominated cooling channel. Alternatively, if a thermal pulse occurs as the star evolves off the AGB, much of the remaining hydrogen is incinerated and nuclear processed material from deeper layers (e.g. $\mathrm{C}$ and $\mathrm{O}$ ) is dredged up to the surface leading to a hydrogen deficient object (e.g. Iben et al. 1983; Herwig et al. 1999).

A number of empirical results are broadly supportive of this hypothesis. For example, the observed number ratios of hydrogen rich to hydrogen-deficient central stars of planetary nebulae (2:1; Mendez 1991) and DAs to DOs at $T_{\text {eff }} \gtrsim 40000 \mathrm{~K}$ (7:1; Fleming et al. 1986) are roughly consistent with theoretical expectations (Iben \& Tutukov 1984). The observed transformation of the hydrogen deficient PG 1159 central star of the planetary nebula, Lo4, into a Wolf Rayet object and back

* Based on observations collected at the European Southern Observatory, Chile. ESO No. 072.D-0362. over a period of months (Werner et al. 1992) and similarities in the measured abundances of the elements $\mathrm{C}$ and $\mathrm{O}$ in the atmospheres of Wolf Rayet $(\mathrm{C} / \mathrm{He} \sim 0.2$ and $\mathrm{O} / \mathrm{He} \sim 0.05$, Koesterke \& Hamann 1997) and PG 1159 stars (C/He $0.1-0.6$ and $\mathrm{O} / \mathrm{He} \sim 0.005-0.1$, Dreizler \& Heber 1998) confirm an evolutionary link between these two classes of object. Furthermore, the abundance patterns observed in the atmospheres of the DO white dwarfs $\left(120000 \gtrsim T_{\text {eff }} \gtrsim 45000 \mathrm{~K}\right.$, $\mathrm{C} / \mathrm{He} \sim 0.001-0.01, \mathrm{O} / \mathrm{He} \lesssim 0.001$; Dreizler 1998) suggest they are the descendents of the PG 1159 stars $\left(180000 \gtrsim T_{\text {eff }} \gtrsim\right.$ $65000 \mathrm{~K}$ ), gravitational settling having reduced the abundances in the former.

Despite detecting $\sim 110$ hot DA white dwarfs $\left(T_{\text {eff }} \gtrsim\right.$ $25000 \mathrm{~K})$, the extreme-ultraviolet surveys of the ROSAT WFC and EUVE unearthed only one new DO white dwarf, RE J0503-285. An analysis of IUE and Voyager data and the optical identification spectrum revealed an effective temperature and surface gravity of $T_{\text {eff }} \approx 70000 \mathrm{~K}$ and $\log g=$ 7.5-8.0 respectively (Barstow et al. 1994). These values are consistent with more recent determinations utilising more refined synthetic spectra and/or higher $\mathrm{S} / \mathrm{N}$ optical data $\left(T_{\text {eff }}=\right.$ $72660_{-6289}^{+2953} \mathrm{~K}, \log g=7.50_{-0.15}^{+0.13}$, Barstow et al. 2000; $T_{\text {eff }}=$ $70000 \mathrm{~K}, \log g=7.50$, Dreizler \& Werner 1996).

Somewhat counter-intuitively to it's detection as an EUV source, of the 14 DOs studied in detail by Dreizler \& Werner (1996) and Dreizler (1998), RE J0503-318 is one of the most metal rich, with $\mathrm{C} / \mathrm{He} \approx 0.005, \mathrm{O} / \mathrm{He} \approx 0.0005, \mathrm{~N} / \mathrm{He} \approx 10^{-5}$ 
Table 1. Summary details of RE J0503-285, including coordinates and near-IR magnitudes obtained from the 2MASS All-Sky Point Source Catalogue. The exposure times used for the acquisition of the $J H$ and $H K$ near-IR spectra with the NTT and SOFI are also listed.

\begin{tabular}{ccccccccc}
\hline \hline Identity & Name & RA & Dec & $J$ & $H$ & $K_{\mathrm{S}}$ & \multicolumn{2}{c}{$t_{\text {exp }}(\mathrm{s})$} \\
& & \multicolumn{2}{c}{$\mathrm{J} 2000.0$} & & & $J H$ & $H K$ \\
\hline WD 0501-289 & RE J0503-285 & 050355.51 & -285434.6 & $14.65 \pm 0.03$ & $14.77 \pm 0.06$ & $14.95 \pm 0.14$ & 1080 & 2880 \\
\hline
\end{tabular}

and $\mathrm{Si} / \mathrm{He} \approx 10^{-5}$. Nickel has also been detected in the photosphere at an abundance of $\mathrm{Ni} / \mathrm{H} \approx 10^{-5}$ (Barstow et al. 2000). However, no convincing evidence of the presence of iron is found in any of the spectral datasets obtained to date, setting an upper limit on its abundance of $\mathrm{Fe} / \mathrm{H} \lesssim 10^{-6}$. To an extent this is an intriguing result when one notes that the cosmic abundance ratio of $\mathrm{Fe}: \mathrm{Ni}$ is $18: 1$, that $\mathrm{Fe}$ is observed to be more abundant than $\mathrm{Ni}$ in the atmospheres of hot DA white dwarfs by factors between 1 to 20 and that self-consistent model calculations taking into account gravitational settling and radiative levitation predict an apparent Fe:Ni ratio of $\sim 1: 3$ (e.g. Dreizler 1998). Barstow \& Sion (1994) report evidence of episodic massloss in a series of IUE spectra of RE J0503-285. It is plausible that the observed pattern of abundances may be reflective of this process. Alternatively, the abundance pattern may be related to the hypothetical dredging up of nuclear processed material from deeper layers caused by the late-thermal pulse, as has been proposed to explain the Fe deficiency observed in a number of PG1159 stars (e.g. Miksa et al. 2002). However, as the mass of RE J0503-285 maybe somewhat lower than the canonical DO value of $0.59 \pm 0.08 M_{\odot}$ (Dreizler \& Werner 1996) it is a possibility that binary evolution (e.g. Vennes et al. 1998) has influenced or continues to interfere with the photospheric composition. Indeed, as discussed by Bragaglia et al. (1990) it is possible that close binary evolution produces preferentially non-DA white dwarfs with hydrogen-deficient envelopes.

The lack of any prior published detailed examination of this particularly interesting object at near-IR wavelengths has motivated us to obtain, during a recent study of DA white dwarfs, a $J H K$ spectrum of RE J0503-285 to investigate external factors possibly influencing its evolution and photospheric composition e.g. the presence of a cool low mass companion. We present here the results of our analysis of this data.

\section{Observations}

\subsection{Data acquisition}

A low resolution near-IR spectrum of RE J0503-285 was obtained using the ESO New Technology Telescope (NTT) and the Son-of-Isaac (SOFI) infrared instrument on 2003/12/10. The sky conditions at the La Silla site were good on this night with seeing typically in the range $0.6^{\prime \prime}-1.0^{\prime \prime}$. We used the low resolution spectroscopic mode $\left(\lambda / \delta \lambda \sim 950\right.$ with the $0.6^{\prime \prime}$ slit), in which coverage of the wavelength ranges $0.95-1.64 \mu \mathrm{m}$ and $1.53-2.52 \mu \mathrm{m}$ is provided by the "blue" and the "red" grism respectively. The observations were undertaken using the standard technique of nodding our point source target back and forth along the spectrograph slit in an ABBA pattern. The total integration times used for the blue and the red grism setup were $1080 \mathrm{~s}$ and $2880 \mathrm{~s}$ respectively. To facilitate the removal of telluric features from the target spectra and to provide an approximate flux calibration, a standard star (HIP 28999) was observed immediately after the science integrations. This was carefully chosen to lie within $\sim 0.1$ airmasses of the white dwarf.

\subsection{Data reduction}

We have used software routines in the STARLINK packages KAPPA and FIGARO to apply standard reduction techniques to our data. Further details of these procedures are given in Dobbie et al. (2005). Here we re-iterate that any features intrinsic to the energy distribution of the standard star were identified by reference to a near-IR spectral atlas of fundamental MK standards (Wallace et al. 2000; Meyer et al. 1998; Wallace \& Hinkle 1997) and were removed by linearly interpolating over them. Furthermore, the flux levels of the data were scaled to (1) achieve the best possible agreement between the blue and the red spectrum of the white dwarf in the overlap region between 1.53-1.64 $\mu \mathrm{m}$ and (2) obtain the best possible agreement between the spectral data and the $J, H$ and $K_{\mathrm{S}}$ photometric fluxes for RE J0503-285 derived from the 2MASS All Sky Data Release Point Source Catalogue magnitudes (Skrutskie et al. 1997) where zero magnitude fluxes were taken from Zombeck (1990). The reduced spectrum and 2MASS fluxes are shown in Fig. 1.

\section{Analysis of the data}

\subsection{Model DO white dwarf spectra}

We have generated a $\mathrm{He}+\mathrm{C}+\mathrm{H}$ synthetic white dwarf spectrum with abundances $\mathrm{C} / \mathrm{He}=0.005$ and $\mathrm{H} / \mathrm{He}=10^{-5}$, at the effective temperature and surface gravity shown in Table 2 . We have used the latest versions of the plane-parallel, hydrostatic, non-local thermodynamic equilibrium (non-LTE) atmosphere and spectral synthesis codes TLUSTY (v200; Hubeny 1988; Hubeny \& Lanz 1995) and SYNSPEC (v48; Hubeny, I., \& Lanz, T., private communication). The calculation included a full treatment of line blanketing and used state-of-the-art model atoms. In brief, the HeII ion incorporated the 19 lowest energy levels, where the dissolution of the higher lying levels was treated by means of the occupation probability formalism of Hummer \& Mihalas (1988), generalised to the non-LTE situation by Hubeny et al. (1994). Lines originating from the four lowest levels were treated by means of an approximate Stark profile (Hubeny et al. 1994) during the calculation of the model 


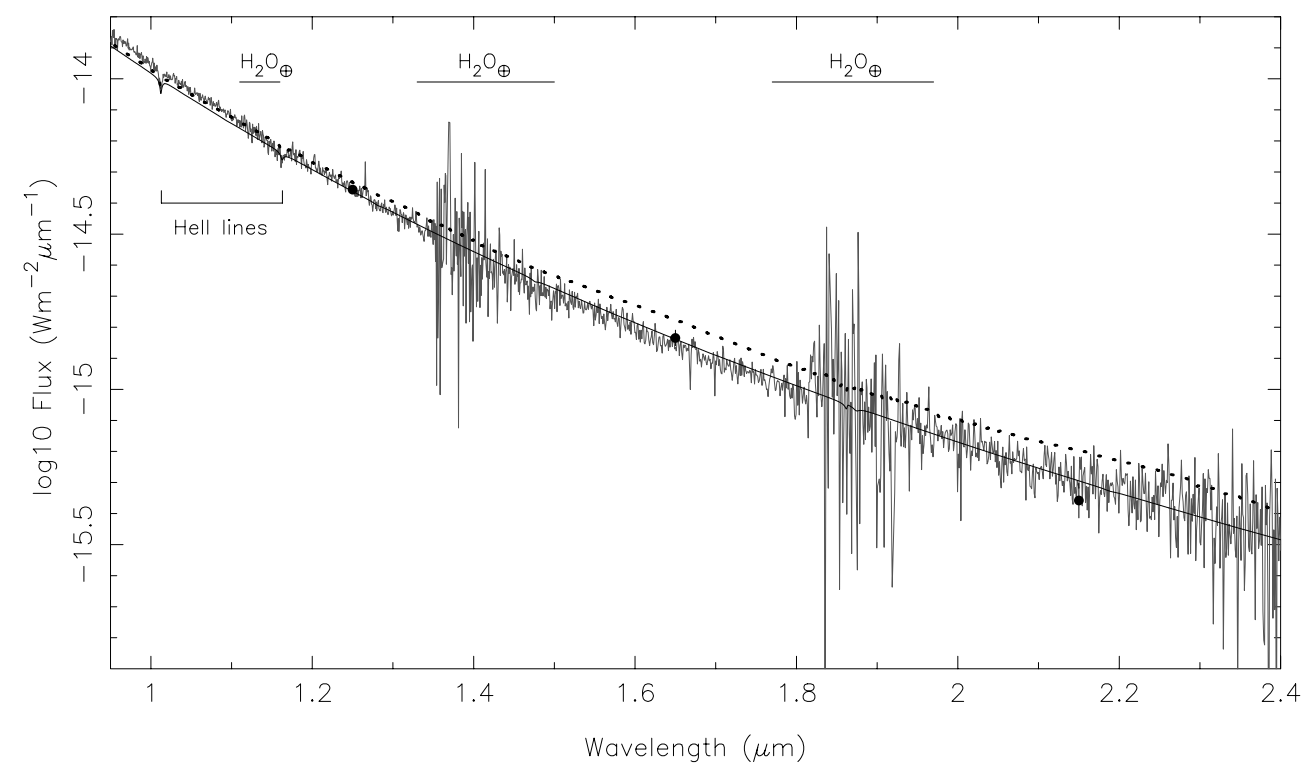

Fig. 1. Near-IR spectroscopy (solid grey line) and 2MASS $J H K$ photometry (filled circles) of the white dwarf RE J0503-285. A He+C+H non-LTE synthetic white dwarf spectrum of appropriate effective temperature, surface gravity and normalisation (solid black line) and a hybrid white dwarf + late-type dwarf model representing our estimated limit on the spectral type of a putative spatially unresolved companion (dotted black line, WD+M8) are overplotted. We have labelled the more prominent white dwarf HeII lines and telluric water vapour features present in this dataset.

Table 2. Summary of additional physical parameters of RE J0503285 taken from Barstow et al. (1994, 2000). Shown in brackets are the values of effective temperature and surface gravity, lying within the uncertainties of Barstow et al.'s analysis, which place RE J0503285 at the largest likely distance. We adopt this latter value in setting a limit on the spectral type of a putative cool companion. Note, the distances have been estimated using pure-C core evolutionary models of Wood (1995) which exclude a hydrogen layer.

\begin{tabular}{cccc}
\hline \hline$T_{\text {eff }}(\mathrm{K})$ & $\log g$ & $V$ & $D(\mathrm{pc})$ \\
\hline $72660(75613)$ & $7.50(7.35)$ & $13.90 \pm 0.01$ & $152(176)$ \\
\hline
\end{tabular}

structure and in the spectral synthesis step. The CIV ion incorporated levels up to $n=14$ where levels $9 \leq n \leq 14$ were each represented by a superlevel. Where available, photoionization cross sections were obtained from TOPbase. Transitions between the lowest level and those with $n \leq 5$ were represented by Stark profiles.

The synthetic energy distribution has been normalised to the $V$ magnitude of RE J0503-285 and convolved with a Gaussian to match the resolution of the SOFI spectrum. This is shown overplotted on the observed data in Fig. 1. It is worth noting here that our modelling indicates that at the effective temperature of this white dwarf, the colours $V-K, J-H$ and $H-K$ are rather weak functions of $T_{\text {eff }}$.

\subsection{The search for a cool companion}

We have examined Fig. 1 for significant differences in the overall shape or level between the observed and synthetic fluxes which can be consistent with the presence of a cool companion. Further, we have searched for specific features in the spectrum typical of the energy distributions of M or L dwarfs e.g. K I and
$\mathrm{Na}$ I absorption at $1.25 \mu \mathrm{m}$ and $2.20 \mu \mathrm{m}$ respectively, $\mathrm{CO}$ at $2.3 \mu \mathrm{m}$ and $\mathrm{H}_{2} \mathrm{O}$ centred on $1.15,1.4$ and $1.9 \mu \mathrm{m}$. However, no convincing evidence for such has been found. Hence, we have added empirical models for low mass stellar and substellar objects, full details of which are given in Dobbie et al. (2005), to the white dwarf synthetic spectrum and compared these composites to the IR data to set an approximate limit on the spectral type of a putative cool companion.

The fluxes of the empirical models have been scaled to a level appropriate to a location at $d=10 \mathrm{pc}$ using the 2MASS $J$ magnitude of each late-type object and the polynomial fits of Dahn et al. (2002) and Tinney et al. (2003) to the $M_{J}$ versus spectral type for M6-M9 and L0-L8 field dwarfs/brown dwarfs respectively. These fluxes have been further reduced by a factor 1.4, corresponding to the rms dispersion in the $M_{J}$ versus spectral type relationship of Tinney et al. (2003). Subsequently, the fluxes have been re-calibrated to be consistent with the distance of the DO as derived from the measured $V$ magnitude, the effective temperature and theoretical $M_{V}$ and radius from evolutionary models of pure- $\mathrm{C}$ core white dwarfs which include only a He layer mass of $10^{-4} M_{\odot}$ (Wood 1995). As the effective temperature and surface gravity and hence our distance determination of RE J0503-285 are considerably less well constrained than those of the DA white dwarfs we have previously investigated (e.g. Dobbie et al. 2005), for a conservative limit we have adopted the largest distance estimate consistent with Barstow et al.'s analysis and the error limits given therein (see Table 2). In fact the neglect of line blanketing arising from metals other than carbon present in the atmosphere of RE J0503285 impacts on the temperature estimate in a such a way as to reinforce our cautious approach (e.g. see Barstow et al. 2000). Starting with L8 we have progressively added earlier spectral types to the synthetic white dwarf spectrum, until it could be 
Table 3. Limiting spectral type, temperature and mass of a putative cool companion to RE J0503-285. The effective temperature is estimated from the polynomial relation detailed in Table 4 of Golimowski et al. (2004). Furthermore, we provide rough upper limits on the mass of a putative cool companion as a function of age, by comparing this effective temperature to the predictions of the evolutionary models of Baraffe et al. (2003).

\begin{tabular}{rrrrr}
\hline \hline SpT & $T_{\text {eff }}(\mathrm{K})$ & $1 \mathrm{Gyr}\left(M_{\odot}\right)$ & $5 \mathrm{Gyr}\left(M_{\odot}\right)$ & $10 \mathrm{Gyr}\left(M_{\odot}\right)$ \\
\hline $\mathrm{M} 8$ & 2500 & 0.084 & 0.085 & 0.085 \\
\hline
\end{tabular}

concluded with reasonable certainty that the presence of a companion of that effective temperature or greater would have been obvious from our data, given the $\mathrm{S} / \mathrm{N}$.

\section{Results}

\subsection{A limit on the mass of a pututive companion to RE J0503-285}

The effective temperatures of very-low-mass stars and substellar objects remain a function of both mass and age on the timescale typical of the lifetime of the white dwarfs likely progenitor ( $\sim \mathrm{Gyr})$. Although we can estimate the cooling age of RE J0503-285 using theoretical evolutionary models, as we don't know with any certainty the mass and hence lifetime of its progenitor star (the initial-mass final-mass relationship is poorly constrained for white dwarfs of this mass and in any case may not apply to DOs) a robust estimate of the age of a putative associate of the DO is not possible. Therefore, to use our limit on spectral-type to constrain mass, we instead assume a range of ages broadly encompassing the likely value. We assign an approximate effective temperature to the spectral type limit shown in Table 3 using the polynomial fit described in Table 4 of Golimowski et al. (2004). Subsequently, we refer to the low mass stellar/substellar evolutionary models for solar metallicity of Baraffe et al. (2003), using cubic splines to interpolate between their points, to estimate corresponding mass at ages 1 Gyr, 5 Gyr and 10 Gyr as shown in Table 3.

We note that there are a number of examples of post common envelope white dwarf + main sequence binaries, where a low mass companion has survived a phase of common envelope evolution e.g. RE J0720-318, RE J1016-05, and RE J2013+400 and now interacts with the white dwarf to influence the composition of its photosphere. Our result argues strongly against the presence of an unresolved cool companion to this white dwarf with $M \gtrsim 0.085 M_{\odot}$ and we can now rule out that the pattern of abundances observed in the atmosphere of RE J0503-285 is influenced by ongoing interaction with such an object. However, our result does not exclude the possibility that the evolution of RE J0503-285 was affected by the interaction of the white dwarf's progenitor star and a close companion $\left(M \gtrsim 0.085 M_{\odot}\right)$ during a common envelope phase from which the latter failed to emerge. Further, there remains the possibility of a close companion with a mass below our detection threshold. This may seem unlikely given that an extensive near-IR study of a sample of 371 white dwarfs, summarised by Farihi et al. (2005), has revealed only two secondaries with spectral types later than
M, GD165B (L4; Becklin \& Zuckerman 1998) and GD1400B (L6; Farihi \& Christopher 2004; Dobbie et al. 2005), the former at a separation a $\sim 120$ AU from its white dwarf primary. Nevertheless, there are persistent although perhaps controversial claims for L dwarf secondaries in some cataclysmic variables such as EF Eri (e.g. Howell \& Ciardi 2001; Harrison et al. 2003).

\subsection{Possible future work}

We point out that RE J0503-285 and the other DO white dwarfs are potential targets for Spitzer imaging to search for a mid-IR excess due to companions cooler than spectral type M. Our non-LTE model of RE J0503-285 indicates the white dwarf flux at $10 \mu \mathrm{m}$ to be $\approx 0.04 \mathrm{mJy}$. From the Spitzer spectrum of DENIS-P J0255-4700 (Roellig et al. 2004), which we determine to reside at $d=5.3 \mathrm{pc}$ using the 2MASS magnitude $(J=13.25 \pm 0.03)$ and the $M_{J}$ versus spectral type relationship of Tinney et al. (2003), allowing for a $25 \%$ uncertainty in the absolute calibration of the mid-IR data and the uncertainties in late-type dwarf fluxes and the white dwarf distance discussed in Sect. 3.2, we estimate the flux level of an L8 companion to RE J0503-285 to be $\approx 0.004 \mathrm{mJy}$. As the absolute accuracy of Spitzer photometry is expected to be $\sim 5 \%$, it should be possible to extend this search to the regime of the coolest L dwarfs, corresponding to substellar masses. Further, improved line broadening theories specifically tailored to helium plasmas would offer the possibility of reducing the uncertainties on our estimates of the effective temperatures and surface gravities of DO white dwarfs and allow more robust determinations of their distances.

Acknowledgements. P.D.D., M.R.B., A.N.L. and R.N. are supported by PPARC. This publication makes use of data products from the Two Micron All Sky Survey, which is a joint project of the University of Massachusetts and the Infrared Processing and Analysis Center/California Institute of Technology, funded by the National Aeronautics and Space Administration and the National Science Foundation. We thank the anonymous referee for useful comments which have improved this work.

\section{References}

Baraffe, I., Chabrier, G., Barman, T. S., Allard, F., \& Hauschildt, P. H. 2003, A\&A, 402, 701

Barstow, M. A., \& Sion, E. M. 1994, MNRAS, 271, L52

Barstow, M. A., Holberg, J. B., Werner, K., Buckley, D. A. H., \& Stobie, R. S. 1994, MNRAS, 267, 653

Barstow, M. A., Dreizler, S., Holberg, J. B., et al. 2000, MNRAS, 314, 109

Becklin, E., \& Zuckerman, B. 1988, Nature, 336, 656

Bragaglia, A., Greggio, L., Renzini, A., \& D’Odorico, S. 1990, ApJ, 365, L13

Dahn, C., Harris, H. C., Urba, F. J., et al. 2002, AJ, 124, 1170

Dobbie, P. D., Burleigh, M. R., Levan, A. J., et al. 2005, MNRAS, 357, 1049

Dreizler, S. 1999, A\&A, 352, 632

Dreizler, S., \& Werner, K. 1996, A\&A, 314, 217

Dreizler, S., \& Heber, U. 1998, A\&A, 334, 618

Dupuis, J., Fontaine, G., Pelletier, C., \& Wesemael, F. 1993, ApJS, 84, 73 
Farihi, J., \& Christopher, M. 2004, AJ, 128, 1868

Farihi, J., Becklin, E. E., \& Zuckerman, B. 2005, ApJS, in press Fleming, T., Liebert, J., \& Green, R. F. 1986, ApJ, 308, 176

Golimowski, D. A., Leggett, S. K., Marley, M. S., et al. 2004, AJ, 127, 3516

Harrison, T. E., Howell, S. B., Szkody, P., et al. 2004, AJ, 614, 947

Herwig, F., Blocker, T., Langer, N., \& Driebe, T. 1999, A\&A, 349, 5

Howell, S. B., \& Ciardi, D. R. 2001, ApJ, 550, L57

Hubeny, I. 1988, Comput. Phys. Commun., 52, 103

Hubeny, I., Hummer, D., \& Lanz, T. 1994, A\&A, 282, 151

Hubeny, I., Lanz, T., \& Jeffrey, C. S. 1994, in Newsletter on Analysis of Astron. Spe., ed. C. S. Jeffrey, 30

Hubeny, I., \& Lanz, T. 1995, ApJ, 439, 875

Hummer, D., \& Mihalas, D. 1988, ApJ, 331, 794

Iben, I., \& Tutukov, A. 1984, ApJ, 277, 333

Iben, I., Kaler, J. B., Truran, J. W., \& Renzini, A. 1983, ApJ, 264, 605

Koesterke, L., \& Hamann, W.-R. 1997, A\&A, 320, 91

Lemke, M. 1997, A\&AS, 122, 285

Mendez, R. H. 1991, in Evolution of Stars: the Photospheric Abundance Connection: Proc. of the 145th Symposium of the International Astronomical Union, ed. G. Michaud, \& A. V. Tutukov (Dordrecht: Kluwer), IAU Symp., 145, 375
Meyer, M. R., Edwards, S., Hinkle, K. H., \& Strom, S. E. 1998, ApJ, 508, 397

Miksa, S., Deetjen, J. L., Dreizler, S., et al. 2002, A\&A, 389, 953

Rauch, T., Heber, U., Hunger, K., Werner, K., \& Neckel, T. 1991, A\&A, 241, 457

Roellig, T. L., Van Cleve, J. E., Sloan, G. C., et al. 2004, ApJS, 154, 418

Schatzman, E. 1958, in White Dwarfs (North Holland Publishing Company)

Skrutskie, M. F., et al. 1997, in The Impact of Large Scale Near-IR Sky Surveys, ed. F. Garzon et al. (Dordrecht: Kluwer), 25

Tinney, C. G., Burgasser, A.J., \& Kirkpatrick, J. D. 2003, AJ, 126, 975

Vennes, S., Dupuis, J., Chayer, P., et al. 1998, ApJ, 500, L41

Wallace, L., \& Hinkle, K. 1997, ApJ, 111, 445

Wallace, L., Meyer, M. R., Hinkle, K., \& Edwards, S. 2000, ApJ, 535, 325

Werner, K., Hamann, W.-R., Heber, U., et al. 1992, A\&A, 259, L69

Wood, M. A. 1995, in Lecture Notes in Physics, White Dwarfs, ed. D. Koester, \& K. Werner (Berlin: Springer), 41

Zombeck, M. V. 1990, Handbook of Astronomy \& Astrophysics, 2nd ed. (UK: Cambridge Univ. Press) 